\title{
A FAST ITERATIVE METHOD TO COMPUTE THE FLOW AROUND A SUBMERGED BODY
}

\author{
JOHAN F. MALMLIDEN AND N. ANDERS PETERSSON
}

\begin{abstract}
We develop an efficient iterative method for computing the steady linearized potential flow around a submerged body moving in a liquid of finite constant depth. In this paper we restrict the presentation to the twodimensional problem, but the method is readily generalizable to the threedimensional case, i.e., the flow in a canal. The problem is indefinite, which makes the convergence of most iterative methods unstable. To circumvent this difficulty, we decompose the problem into two more easily solvable subproblems and form a Schwarz-type iteration to solve the original problem. The first subproblem is definite and can therefore be solved by standard iterative methods. The second subproblem is indefinite but has no body. It is therefore easily and efficiently solvable by separation of variables. We prove that the iteration converges for sufficiently small Froude numbers. In addition, we present numerical results for a second-order accurate discretization of the problem. We demonstrate that the iterative method converges rapidly, and that the convergence rate improves when the Froude number decreases. We also verify numerically that the convergence rate is essentially independent of the grid size.
\end{abstract}

\section{INTRODUCTION}

The subject of this paper is an efficient, Schwarz-type, iterative method for computing the steady linearized potential flow around a submerged body moving in a liquid of finite constant depth. Let the depth of the liquid be $d$, the speed of the body be $U$ and the acceleration of gravity be $g$. After scaling the physical quantities by the length $d$ and the velocity $\sqrt{g d}$, we get the problem depicted in Figure 1 . The total velocity potential is split into the sum of a free stream potential and a perturbation potential; $\Phi(x, z)=\mu x+\phi(x, z)$, where $\mu=U / \sqrt{g d}$ is the Froude number. The perturbation potential is governed by, cf. [18, pp. 431-448],

$$
\Delta \phi=0, \quad-\infty<x<\infty,-1<z<0,
$$

together with the boundary conditions

Received by the editor March 20, 1992 and, in revised form, August 9, 1994.

1991 Mathematics Subject Classification. Primary 65N12, 76B20.

Key words and phrases. Schwarz iteration, finite difference approximation, composite overlapping grid, potential flow.

The first author was partially supported by ONR grant N-00014-90-J-1382 and by the U.S. Department of Energy through Los Alamos National Laboratory.

The second author was supported by ONR grants N-00014-90-J-1695, N-00014-90-J-1382 and by the U.S. Department of Energy through Los Alamos National Laboratory.

(c)1996 American Mathematical Society 


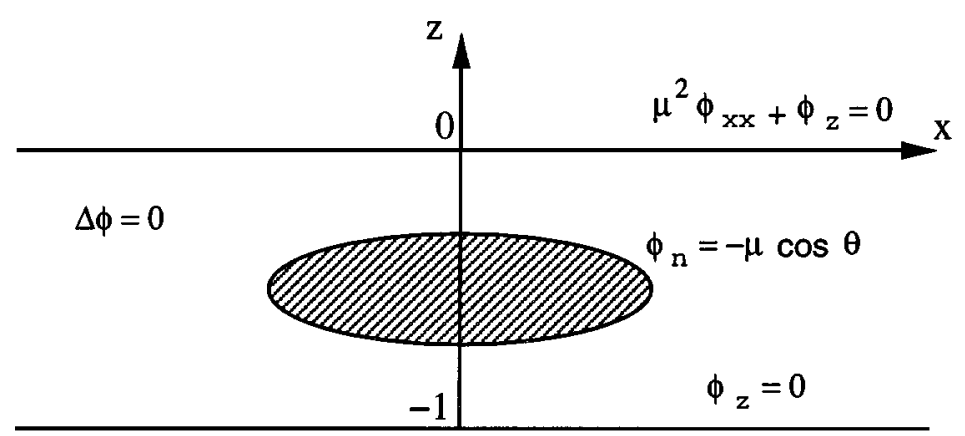

Figure 1. The linearized problem

$$
\begin{aligned}
\mu^{2} \phi_{x x}+\phi_{z} & =0, & & -\infty<x<\infty, z=0, \\
\phi_{z} & =0, & & -\infty<x<\infty, z=-1, \\
\partial \phi / \partial n+\mu \cos \theta & =0, & & \text { on the body. }
\end{aligned}
$$

Here, $\partial / \partial n$ denotes the outward normal derivative and $\theta$ is the angle between the normal and the $x$-axis. We are looking for a solution where the perturbation potential tends to zero at large distances in front of the body. This condition is called the upstream condition,

$$
\lim _{x \rightarrow-\infty} \phi=0, \quad-1<z<0 .
$$

Three main classes of numerical techniques have previously been used to solve the present problem. These are based on boundary integral methods, finite element techniques and finite difference formulations. There are two different types of boundary integral methods. The first uses a kernel which satisfies the boundary condition on the free surface and the upstream condition, cf. [7, 14]. In this method, there are only dependent variables along the surface of the body. However, the kernel is rather difficult and expensive to evaluate numerically [15]. The second boundary integral method employs a kernel which does not satisfy any boundary conditions but is easy and inexpensive to evaluate, cf. $[6,9,20]$. Here, the dependent variables are located along the boundary of the body and on the infinite surface, which needs to be truncated in the numerical approximation. A number of implementations of these two boundary integral approaches were competitively compared to towing-tank experimental data in [11]. The finite element techniques are based on dividing the fluid domain into two subdomains, one close to the body and one outer domain. The domain close to the body is discretized by finite elements and analytical solutions are used ahead of and behind the body. This approach, called the hybrid or localized finite element method, is pursued in $[3,10,13,19]$. Similar to the finite element technique, the finite difference methods $[16,17]$ divide the infinite domain into one region close to the hull, where the solution is computed numerically, coupled to the outer domain by far-field boundary conditions.

All the previous methods have in common that they must solve the linear system that emanates from the discretization procedure. In the boundary integral method, the matrix is full and has dimension equal to the number of boundary points. In the finite element technique and the finite difference method, the discretization procedure yields a sparse matrix with dimension equal to the number of points in the flow field. Owing to memory and work requirements, it is very expensive to solve these linear systems by a direct method like Gaussian elimination, especially in the 
three-dimensional counterpart of the present problem. The continuous problem (1-3) is indefinite, which implies that also the linear system is indefinite, regardless of the discretization method. This makes the convergence of most iterative methods unstable. To circumvent this difficulty, we propose a novel iterative technique where the problem is decomposed into two more easily solvable subproblems that are coupled by a Schwarz-type iteration such that the original problem is solved upon convergence. In this paper we expound the method for the two-dimensional case, but the technique is readily generalizable to the three-dimensional problem [12].

The first subproblem, which will be referred to as the definite subproblem, is defined by

$$
\Delta \phi^{I}=0,-\infty<x<\infty,-1<z<0,
$$

together with the boundary conditions

$$
\begin{aligned}
\phi_{z}^{I} & =0, & & -\infty<x<\infty, z=0, \\
\phi_{z}^{I} & =0, & & -\infty<x<\infty, z=-1, \\
\partial \phi^{I} / \partial n & =h, & & \text { on the body. }
\end{aligned}
$$

To fix the undetermined constant in this Neumann problem we enforce

$$
\lim _{x \rightarrow-\infty} \phi^{I}=0, \quad-1<z<0 .
$$

The second subproblem, which will be called the indefinite subproblem, does not have a submerged body in the interior of the domain. It is governed by

$$
\Delta \phi^{I I}=0, \quad-\infty<x<\infty,-1<z<0,
$$

subject to the boundary conditions

$$
\begin{aligned}
\mu^{2} \phi_{x x}^{I I}+\phi_{z}^{I I} & =t, & & -\infty<x<\infty, z=0, \\
\phi_{z}^{I I} & =0, & & -\infty<x<\infty, z=-1 .
\end{aligned}
$$

In order to make the solution unique, we enforce the upstream condition,

$$
\lim _{x \rightarrow-\infty} \phi^{I I}=0, \quad-1<z<0 .
$$

The first subproblem is definite and can therefore be solved by standard iterative methods. The second subproblem is indefinite but has no body. It is therefore easily and efficiently solvable by separation of variables. That solution method will be described in $\S 2$.

The solutions of the subproblems are well defined once the forcing functions $h$ and $t$ are determined. It is clear that $\phi^{I}+\phi^{I I}$ will solve (1-3) if we can find functions $t$ and $h$ that simultaneously satisfy $t(x)=-\mu^{2} \phi_{x x}^{I}(x, 0)$ and $h(s)=$ $-\mu \cos \theta(s)-\partial \phi^{I I} / \partial n\left(x_{b}(s), z_{b}(s)\right)$, where the boundary of the body is described by $x=x_{b}(s), z=z_{b}(s), 0 \leq s \leq 1$. We compute $t$ and $h$ by iteration. We take the initial guess to be $\phi^{I I(0)}(x, z) \equiv 0$ and iterate according to

1. Set $h^{(i)}(s)=-\mu \cos \theta(s)-\partial \phi^{I I(i-1)} / \partial n\left(x_{b}(s), z_{b}(s)\right)$ and solve the definite subproblem for $\phi^{I(i)}$.

2. Set $t^{(i)}(x)=-\mu^{2} \phi_{x x}^{I(i)}(x, 0)$, and solve the indefinite subproblem for $\phi^{I I(i)}$.

The main result of this paper, which is proven in $\S 3$, is that the iteration converges for sufficiently small Froude numbers. In order to demonstrate the convergence numerically, we truncate the infinite domain and introduce far-field boundary conditions in $\S 4$ to carry out the practical computation. In $\S 5$ we present numerical 
results for a second-order accurate discretization of (1-3). We show that the iterative method converges rapidly, and that the convergence rate improves when the Froude number decreases. We also verify numerically that the convergence rate is essentially independent of the grid size. Perhaps more surprising is that the iterative method requires less CPU-time than the direct solver described in [17], already for two-dimensional problems.

\section{ThE INDEFINITE SUBPROBLEM}

To solve the indefinite subproblem, we split the solution according to $\phi^{I I}=$ $\phi^{a}+\phi^{s}$. The idea is to use $\phi^{a}$ to move the inhomogeneity from the surface boundary condition to an inhomogeneity for the Laplace equation and then solve the resulting problem for $\phi^{s}$ by separation of variables. Henceforth, we assume that (9) is satisfied on the boundary $z=0$ and make the substitution $\phi_{x x}^{I I}=-\phi_{z z}^{I I}$ in (10). The auxiliary function $\phi^{a}$ must satisfy

$$
\begin{aligned}
-\mu^{2} \phi_{z z}^{a}+\phi_{z}^{a} & =t, & & -\infty<x<\infty, z=0, \\
\phi_{z}^{a} & =0, & & -\infty<x<\infty, z=-1 .
\end{aligned}
$$

In the interior, $\phi^{a}$ is only required to be smooth. We will use the following simple solution:

$$
\phi^{a}(x, z)=\frac{t(x)}{2\left(1-\mu^{2}\right)}(1+z)^{2} .
$$

In order to make $\phi^{a}+\phi^{s}$ satisfy (9-12), we must have

$$
\Delta \phi^{s}=f, \quad-\infty<x<\infty,-1<z<0,
$$

where $f(x, z)=-\Delta \phi^{a}(x, z)$, together with the boundary conditions

$$
\begin{aligned}
-\mu^{2} \phi_{z z}^{s}+\phi_{z}^{s} & =0, & & -\infty<x<\infty, z=0, \\
\phi_{z}^{s} & =0, & & -\infty<x<\infty, z=-1 .
\end{aligned}
$$

To separate variables, we make the ansatz

$$
\phi^{s}(x, z)=\sum_{k=0}^{\infty} R^{(k)}(x) S^{(k)}(z),
$$

where

$$
\begin{aligned}
& S^{(0)}(z)=1, \\
& S^{(1)}(z)=\cosh \sqrt{\lambda}(1+z), \\
& S^{(k)}(z)=\cos \sqrt{\kappa_{k}}(1+z), \quad k=2,3, \ldots .
\end{aligned}
$$

By studying $d S^{(k)} / d z$, cf. [16], it is easy to see that $\left\{S^{(k)}\right\}$ form a complete set in $\mathcal{L}_{2}[-1,0]$. Furthermore, they are orthogonal in the sense

$$
\int_{-1}^{0} \frac{d S^{(p)}}{d z} \frac{d S^{(q)}}{d z} d z=0, p \neq q .
$$

The eigenvalues are given by the relations

$$
\mu^{2} \sqrt{\lambda}=\tanh \sqrt{\lambda}, \mu^{2} \sqrt{\kappa_{k}}=\tan \sqrt{\kappa_{k}}, \quad k=2,3, \ldots
$$

Henceforth, we assume that $0<\mu<1$. This implies a real $\sqrt{\lambda}$. The functions $\cosh z$ and $\cos z$ are symmetric in $z$; we will therefore only consider positive $\sqrt{\lambda}$ and $\sqrt{\kappa_{k}}$. 
If we enter the ansatz (17) into (15) and expand the right-hand side according to

$$
f(x, z)=\sum_{k=0}^{\infty} \hat{f}^{(k)}(x) S^{(k)}(z),
$$

we arrive at the following system of ordinary differential equations:

$$
\begin{aligned}
\frac{d^{2} R^{(0)}}{d x^{2}} & =\hat{f}^{(0)}(x), \\
\frac{d^{2} R^{(1)}}{d x^{2}}+\lambda R^{(1)} & =\hat{f}^{(1)}(x), \\
\frac{d^{2} R^{(k)}}{d x^{2}}-\kappa_{k} R^{(k)} & =\hat{f}^{(k)}(x), \quad k=2,3, \ldots .
\end{aligned}
$$

Next, we express the functions $\hat{f}^{(k)}(x)$ in terms of $t(x)$. To clarify the notation, we define $\zeta^{(k)}:=d S^{(k)} / d z, k=1,2,3, \ldots$ We also define the inner product and norm in the $z$-direction,

$$
\langle a, b\rangle_{z}=\int_{-1}^{0} \bar{a} b d z, \quad\|a\|_{z}^{2}=\langle a, a\rangle_{z} .
$$

By differentiating (21) in the $z$-direction we get

$$
f_{z}(x, z)=\sum_{k=1}^{\infty} \hat{f}^{(k)}(x) \zeta^{(k)}(z) .
$$

The orthogonality relation (19) yields

$$
\hat{f}^{(k)}(x)=\frac{\left\langle f_{z}(x, \cdot), \zeta^{(k)}\right\rangle_{z}}{\left\|\zeta^{(k)}\right\|_{z}^{2}}, \quad k=1,2,3, \ldots
$$

By inserting (14) into $f_{z}(x, z)=-\Delta \phi_{z}^{a}(x, z)$ and evaluating the scalar products and norms analytically, we get

$$
\begin{aligned}
\hat{f}^{(1)}(x) & =\frac{-4 t^{\prime \prime}(x)}{1-\mu^{2}} \cdot \frac{\sinh \sqrt{\lambda}-\sqrt{\lambda} \cosh \sqrt{\lambda}}{2 \lambda^{3 / 2}-\lambda \sinh 2 \sqrt{\lambda}} \\
& =\frac{-2 t^{\prime \prime} \cosh \sqrt{\lambda}}{\lambda\left(\mu^{2} \cosh ^{2} \sqrt{\lambda}-1\right)}, \\
\hat{f}^{(k)}(x) & =\frac{-4 t^{\prime \prime}(x)}{1-\mu^{2}} \cdot \frac{\sin \sqrt{\kappa_{k}}-\sqrt{\kappa_{k}} \cos \sqrt{\kappa_{k}}}{2 \kappa_{k}^{3 / 2}-\kappa_{k} \sin 2 \sqrt{\kappa_{k}}} \\
& =\frac{2 t^{\prime \prime} \cos \sqrt{\kappa_{k}}}{\kappa_{k}\left(1-\mu^{2} \cos ^{2} \sqrt{\kappa_{k}}\right)} .
\end{aligned}
$$

We cannot use the same technique to evaluate $\hat{f}^{(0)}$, because $d S^{(0)} / d z \equiv 0$. Instead we use (21) and compute $\hat{f}^{(0)}$ once the other coefficients are known, i.e.,

$$
\hat{f}^{(0)}(x)=f(x, z)-\sum_{k=1}^{\infty} \hat{f}^{(k)}(x) S^{(k)}(z) .
$$

This equation is valid for all values of $z$, but the choice $z=-1$ makes the occurring expressions particularly simple. By inserting (28) and (29) we get

$$
\hat{f}^{(0)}(x)=-\frac{t(x)}{1-\mu^{2}}-2 t^{\prime \prime}(x) Q
$$


where

$$
Q=\frac{-\cosh \sqrt{\lambda}}{\lambda\left(\mu^{2} \cosh ^{2} \sqrt{\lambda}-1\right)}+\sum_{k=2}^{\infty} \frac{\cos \sqrt{\kappa_{k}}}{\kappa_{k}\left(1-\mu^{2} \cos ^{2} \sqrt{\kappa_{k}}\right)}
$$

\section{Analysis of the iteration}

In this section we prove convergence of the iteration for sufficiently small Froude numbers. The proof consists of estimates of the solutions to the two subproblems. In $\S 3.1$, we estimate the $x$-derivatives of the solution to the definite problem at the surface in terms of the forcing $h$ on the body. Thereafter, in $\S 3.2$, we derive estimates for the $x$ - and $z$-derivatives of the solution to the indefinite problem in terms of the forcing $t$ along the surface. These estimates will be used to bound the normal derivative of the solution to the indefinite problem along the fictitious boundary of the body. These two estimates are combined in $\S 3.3$ to prove convergence of the iteration.

Henceforth, $C$ will denote a generic constant which is independent of $\mu$.

3.1. Estimate for the definite subproblem. To begin with, we define the maximum norm along the boundary of the body according to

$$
|f|_{\infty, \text { body }}=\sup _{0 \leq s \leq 1}\left|f\left(x_{b}(s), z_{b}(s)\right)\right| .
$$

Furthermore, we denote the maximum norm of a function of one or two independent variables by $|\cdot|_{\infty}$.

It is well known, cf. [5], that the $x$-derivatives of $\phi^{I}$ along the surface can be estimated in terms of the forcing $h$. We make this statement more precise in

Lemma 1. There holds

$$
\left|\frac{\partial^{p} \phi^{I(i)}}{\partial x^{p}}(\cdot, 0)\right|_{\infty} \leq C_{p}\left|h^{(i)}\right|_{\infty, \text { body }}, \quad p=1,2, \ldots,
$$

where $C_{p}$ are constants independent of $h^{(i)}$.

In the domains ahead of and behind the body, the solution of the definite subproblem can be found by separation of variables. Let the body be contained in $-\beta \leq x \leq \beta$. Expanding the solution in a Fourier series in the vertical direction yields

$$
\phi^{I}(x, z)=\sum_{k=0}^{\infty} \alpha_{k} e^{-\omega_{k}|x|} \cos \omega_{k} z, \quad \omega_{k}=k \pi,
$$

for $|x|>\beta$. Hence, the forcing function $t(x)$ satisfies

$$
t(x)=\sum_{k=1}^{\infty} \alpha_{k} \omega_{k}^{2} e^{-\omega_{k} x} \leq C e^{-\pi|x|}, \quad|x|>\beta .
$$

3.2. Estimates for the indefinite subproblem. The purpose of this section is to derive bounds for the maximum norm of the $x$ - and $z$-derivatives of $\phi^{I I}$ in terms of the forcing $t$. To bound $\phi^{I I}=\phi^{a}+\phi^{s}$, we bound $\phi^{a}$ and $\phi^{s}$ separately and add the results. Differentiating the solution formula (14) yields 
Lemma 2. We have

$$
\left|\phi_{x}^{a}\right|_{\infty} \leq \frac{\left|t^{\prime}\right|_{\infty}}{2\left(1-\mu^{2}\right)}, \quad\left|\phi_{z}^{a}\right|_{\infty} \leq|t|_{\infty}\left(1-\mu^{2}\right) .
$$

We now consider $\phi^{s}$. We estimate $\phi_{x}^{s}$ and $\phi_{z}^{s}$ in three steps. First we bound the functions $\hat{f}^{(k)}$ in terms of the forcing $t(x)$. Then we estimate the solution of the ordinary differential equations $(22-24)$ in terms of the right-hand sides $\hat{f}^{(k)}$. Finally, we bound $\phi_{x}^{s}$ and $\phi_{z}^{s}$ by summing the bounds for all the modes.

Bounding $\hat{f}^{(k)}$. The eigenvalue relation (20) implies $\sqrt{\lambda}<\mu^{-2}$. In addition, $\sqrt{\lambda} \rightarrow$ $\mu^{-2}$, when $\mu \rightarrow 0$. We have $\mu^{2} \cosh ^{2} \sqrt{\lambda}>1$ for all $0<\mu<1$. Therefore, (28) yields

$$
\left|\hat{f}^{(1)}\right|_{\infty} \leq C \frac{\mu^{2}}{\cosh \mu^{-2}}\left|t^{\prime \prime}\right|_{\infty},
$$

where $C \rightarrow 1$ when $\mu \rightarrow 0$. Inspection of (29) yields directly

$$
\left|\hat{f}^{(k)}\right|_{\infty} \leq \frac{2\left|t^{\prime \prime}\right|_{\infty}}{\kappa_{k}\left(1-\mu^{2}\right)}, \quad k=2,3, \ldots
$$

To bound $\hat{f}^{(0)}$, we need to estimate $Q$ in (31). For the eigenvalues $\sqrt{\kappa_{k}}$ we have

$$
\pi(k-1) \leq \sqrt{\kappa_{k}} \leq \pi(k-1 / 2), \quad k=2,3, \ldots .
$$

Hence,

$$
\sum_{k=2}^{\infty} \kappa_{k}^{-1} \leq \frac{1}{\pi^{2}}\left(1+\int_{1}^{\infty} k^{-2} d k\right)=\frac{2}{\pi^{2}}
$$

Therefore, (30) gives

$$
\left|\hat{f}^{(0)}\right|_{\infty} \leq \frac{C}{1-\mu^{2}}\left(|t|_{\infty}+\left|t^{\prime \prime}\right|_{\infty}\right)
$$

Bounding $R^{(k)}$. We begin by writing down the analytical solutions of (22-24). By integrating (22) twice we find

$$
R^{(0)}(x)=C_{1}+C_{2} x+\int_{-\infty}^{x} \int_{-\infty}^{\zeta} \hat{f}^{(0)}(\xi) d \xi d \zeta .
$$

The upstream condition (12) implies $C_{1}=C_{2}=0$. The general solution of (23) can be written as:

$$
\begin{aligned}
R^{(1)}(x)= & C_{3} e^{i \sqrt{\lambda} x}+C_{4} e^{-i \sqrt{\lambda} x} \\
& +\frac{i}{2 \sqrt{\lambda}} \int_{-\infty}^{x} e^{i \sqrt{\lambda}(\xi-x)} \hat{f}^{(1)}(\xi) d \xi \\
& -\frac{i}{2 \sqrt{\lambda}} \int_{-\infty}^{x} e^{-i \sqrt{\lambda}(\xi-x)} \hat{f}^{(1)}(\xi) d \xi .
\end{aligned}
$$


Again, (12) yields $C_{3}=C_{4}=0$. Finally, the general solution of (24) can be expressed according to

$$
\begin{aligned}
R^{(k)}(x)= & C_{5}^{(k)} e^{\sqrt{\kappa_{k}} x}-C_{6}^{(k)} e^{-\sqrt{\kappa_{k}} x} \\
& -\frac{1}{2 \sqrt{\kappa_{k}}} \int_{x}^{\infty} e^{-\sqrt{\kappa_{k}}(\xi-x)} \hat{f}^{(k)}(\xi) d \xi \\
& -\frac{1}{2 \sqrt{\kappa_{k}}} \int_{-\infty}^{x} e^{\sqrt{\kappa_{k}}(\xi-x)} \hat{f}^{(k)}(\xi) d \xi .
\end{aligned}
$$

By assuming the solution to be bounded at infinity we get $C_{5}^{(k)}=C_{6}^{(k)}=0$.

The decay of $t(x)$ given by (34) implies that the forcing functions $\hat{f}^{(k)}$ will all satisfy

$$
\left|\hat{f}^{(k)}(x)\right| \leq\left|\hat{f}^{(k)}\right|_{\infty} e^{-\pi|x|}, \quad k=0,1,2, \ldots
$$

in $|x|>\beta$.

In the following, the horizontal length of the body will be denoted $L=2 \beta$.

We bound $d R^{(0)} / d x$ by differentiating (41) once. This gives,

$$
\left|\frac{d R^{(0)}}{d x}\right|_{\infty} \leq C L\left|\hat{f}^{(0)}\right|_{\infty}
$$

The solution formula (42) yields the following bounds for $R^{(1)}$ and $d R^{(1)} / d x$ :

$$
\left|R^{(1)}\right|_{\infty} \leq C L \mu^{2}\left|\hat{f}^{(1)}\right|_{\infty}, \quad\left|\frac{d R^{(1)}}{d x}\right|_{\infty} \leq C L\left|\hat{f}^{(1)}\right|_{\infty} .
$$

In the same way, (43) yields the following estimates for $R^{(k)}$ and $d R^{(k)} / d x$ :

$$
\left|R^{(k)}\right|_{\infty} \leq \frac{C L}{\sqrt{\kappa_{k}}}\left|\hat{f}^{(k)}\right|_{\infty}, \quad\left|\frac{d R^{(k)}}{d x}\right|_{\infty} \leq C L\left|\hat{f}^{(k)}\right|_{\infty},
$$

where $k=2,3,4, \ldots$

Bounding $\phi_{x}^{s}$. By differentiating (17) with respect to $x$ we find

$$
\phi_{x}^{s}=\sum_{k=0}^{\infty} \frac{d R^{(k)}(x)}{d x} S^{(k)}(z) .
$$

We combine (40) and (45) to get

$$
\left|\frac{d R^{(0)}}{d x}\right|_{\infty} \leq \frac{C L}{1-\mu^{2}}\left(|t|_{\infty}+\left|t^{\prime \prime}\right|_{\infty}\right) .
$$

The term $d R^{(1)} / d x$ is bounded by using (36) and (46),

$$
\left|\frac{d R^{(1)}}{d x} S^{(1)}(z)\right|_{\infty} \leq \frac{C L \mu^{2}}{\left(1-\mu^{2}\right) \cosh \mu^{-2}}\left|t^{\prime \prime}\right|_{\infty} .
$$

By entering (37) into (47) and using the bound (39) we arrive at

$$
\left|\sum_{k=2}^{\infty} \frac{d R^{(k)}}{d x} S^{(k)}(z)\right|_{\infty} \leq \frac{C L}{1-\mu^{2}}\left|t^{\prime \prime}\right|_{\infty} \sum_{k=2}^{\infty}\left(\kappa_{k}\right)^{-1} \leq \frac{C L}{1-\mu^{2}}\left|t^{\prime \prime}\right|_{\infty} .
$$

Combining (49), (50) and (51) gives 
Lemma 3. There holds

$$
\left|\phi_{x}^{s}(\cdot, z)\right|_{\infty} \leq \frac{C L}{1-\mu^{2}}\left(|t|_{\infty}+\left|t^{\prime \prime}\right|_{\infty}\right) .
$$

Bounding $\phi_{z}^{s}$. To get an equation for $\phi_{z}^{s}$, we differentiate (17) once in the $z-$ direction,

$$
\phi_{z}^{s}(x, z)=\sum_{k=1}^{\infty} R^{(k)}(x) \zeta^{(k)}(z)
$$

By combining (36) and (46) and noting that $\left|\zeta^{(1)}\right| \leq C \mu^{2}$, the first term in the sum satisfies

$$
\left|R^{(1)} \zeta^{(1)}(z)\right|_{\infty} \leq \frac{C L \mu^{2}}{\left(1-\mu^{2}\right) \cosh \mu^{-2}}\left|t^{\prime \prime}\right|_{\infty} .
$$

We get an estimate of the remaining terms by using (37) and (47). This yields,

$$
\left|\sum_{k=2}^{\infty} R^{(k)} \zeta^{(k)}(z)\right|_{\infty} \leq \frac{C L}{1-\mu^{2}}\left|t^{\prime \prime}\right|_{\infty}
$$

By inserting (53) and (54) into (52) we find

Lemma 4. There holds

$$
\left|\phi_{z}^{s}(\cdot, z)\right|_{\infty} \leq \frac{C L}{1-\mu^{2}}\left|t^{\prime \prime}\right|_{\infty}
$$

Bounding $\phi_{x}^{I I}$ and $\phi_{z}^{I I}$. By adding the bound for $\phi_{x}^{a}$ from Lemma 2 and the bound for $\phi_{x}^{s}$ from Lemma 3 , we get

$$
\left|\phi_{x}^{I I}(\cdot, z)\right|_{\infty} \leq \frac{C L}{1-\mu^{2}}\left(|t|_{\infty}+\left|t^{\prime}\right|_{\infty}+\left|t^{\prime \prime}\right|_{\infty}\right) .
$$

Similarly, $\phi_{z}^{I I}$ is estimated by adding the bounds for $\phi_{z}^{a}$ from Lemma 2 and the bound for $\phi_{z}^{s}$ from Lemma 4 ,

$$
\left|\phi_{z}^{I I}(\cdot, z)\right|_{\infty} \leq \frac{C L}{1-\mu^{2}}\left(|t|_{\infty}+\left|t^{\prime \prime}\right|_{\infty}\right) .
$$

Hence, the sum of $\phi_{x}^{I I}$ and $\phi_{z}^{I I}$ is bounded by

$$
\left|\phi_{x}^{I I}(\cdot, z)\right|_{\infty}+\left|\phi_{z}^{I I}(\cdot, z)\right|_{\infty} \leq \frac{C L}{1-\mu^{2}}\left(|t|_{\infty}+\left|t^{\prime}\right|_{\infty}+\left|t^{\prime \prime}\right|_{\infty}\right) .
$$

The definition of the forcing $t(x)$ yields

Lemma 5. We have

$$
\left|\phi_{x}^{I I(i)}(\cdot, z)\right|_{\infty}+\left|\phi_{z}^{I I(i)}(\cdot, z)\right|_{\infty} \leq C L \frac{\mu^{2}}{1-\mu^{2}} \sum_{p=2}^{4}\left|\frac{\partial^{p} \phi^{I(i)}}{\partial x^{p}}(\cdot, 0)\right|_{\infty} .
$$


3.3. Convergence of the iteration. To simplify the notation, we introduce

$$
\begin{aligned}
\Psi^{I(i)}(x, z) & =\phi^{I(i)}(x, z)-\phi^{I(i-1)}(x, z), \\
\Psi^{I I(i)}(x, z) & =\phi^{I I(i)}(x, z)-\phi^{I I(i-1)}(x, z) .
\end{aligned}
$$

We can now prove

Theorem 1. For a sufficiently small $\mu$, the iterative method converges uniformly to $\lim _{i \rightarrow \infty} \phi^{I(i)} \rightarrow \phi^{I}$ and $\lim _{i \rightarrow \infty} \phi^{I I(i)} \rightarrow \phi^{I I}$, where $\phi^{I}+\phi^{I I}$ is a solution of $(1-3)$.

Proof. From Lemma 1 and the definition of $h^{(i)}$ we have

$$
\sum_{p=2}^{4}\left|\frac{\partial^{p} \Psi^{I(i)}}{\partial x^{p}}(\cdot, 0)\right|_{\infty} \leq C\left|h^{(i)}-h^{(i-1)}\right|_{\infty, \text { body }}=C\left|\frac{\partial \Psi^{I I(i-1)}}{\partial n}\right|_{\infty, \text { body }} .
$$

The triangle inequality yields

$$
\begin{aligned}
\left|\frac{\partial \Psi^{I I(i)}}{\partial n}\right|_{\infty, \text { body }} & \leq\left|\Psi_{x}^{I I(i)}\right|_{\infty, \text { body }}+\left|\Psi_{z}^{I I(i)}\right|_{\infty, \text { body }} \\
& \leq\left|\Psi_{x}^{I I(i)}\right|_{\infty}+\left|\Psi_{z}^{I I(i)}\right|_{\infty} .
\end{aligned}
$$

Therefore, Lemma 5 and (60) imply

$$
\begin{aligned}
\left|\frac{\partial \Psi^{I I(i)}}{\partial n}\right|_{\infty, \text { body }} & \leq C L \frac{\mu^{2}}{1-\mu^{2}} \sum_{p=2}^{4}\left|\frac{\partial^{p} \Psi^{I(i)}}{\partial x^{p}}(\cdot, 0)\right|_{\infty} \\
& \leq C L \frac{\mu^{2}}{1-\mu^{2}}\left|\frac{\partial \Psi^{I I(i-1)}}{\partial n}\right|_{\infty, \text { body }} .
\end{aligned}
$$

By choosing $\mu$ so that $\delta=C L \mu^{2} /\left(1-\mu^{2}\right)<1$, the contraction mapping principle ensures uniform convergence, i.e., $\lim _{i \rightarrow \infty} \partial \phi^{I I(i)} / \partial n=\partial \phi^{I I} / \partial n$. Hence, $\lim _{i \rightarrow \infty} h^{(i)}=h$, so $\lim _{i \rightarrow \infty} \phi^{I(i)} \rightarrow \phi^{I}$, and therefore also $\lim _{i \rightarrow \infty} \phi^{I I(i)} \rightarrow \phi^{I I}$. It follows by inspection that $\phi^{I}+\phi^{I I}$ is a solution of (1-3).

\section{FAR-FIELD BOUNDARY CONDITIONS}

It is necessary to bound the computational domain and introduce artificial boundary conditions at the far-field boundaries to carry out the practical calculation. Here, we truncate the domain to $-b<x<b$, see Figure 2. In this section, we will only present the boundary conditions. Their effect on the solution will not be analyzed. We will instead perform numerical experiments to verify that their influence is small if the computational domain is sufficiently large.

For the definite subproblem, we enforce the following artificial boundary conditions:

$$
\begin{aligned}
\phi^{I}(-b, z) & =0, \\
\phi_{x}^{I}(b, z) & =0 .
\end{aligned}
$$

These conditions are local, which makes an iterative solver easy to apply.

In order to solve the indefinite subproblem numerically, we must replace $t(x)$ by a smooth function $\tilde{t}(x):=P(x) t(x)$ which has compact support in the computational 


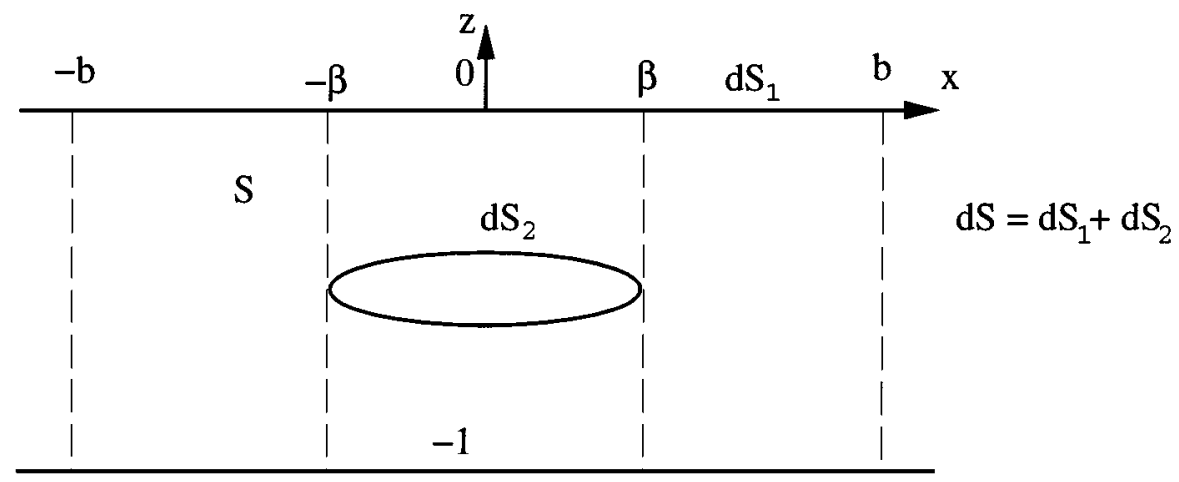

Figure 2. The computational domain

domain. In addition, $P(x)$ must have two continuous derivatives, so that $\tilde{t}^{\prime \prime}$ is well defined. A cutoff function having these properties is given by

$$
P(x)=\left\{\begin{array}{lc}
0, & -\infty<x \leq-b, \\
p_{1}((-b+\alpha-x) / \alpha), & -b<x<-b+\alpha, \\
1, & -b+\alpha \leq x \leq b-\alpha, \\
p_{1}((-b+\alpha+x) / \alpha), & b-\alpha<x<b, \\
0, & b \leq x<\infty,
\end{array}\right.
$$

where $\alpha>0$ and $p_{1}(\xi)=1-10 \xi^{3}+15 \xi^{4}-6 \xi^{5}$. We will denote the solution of $(22-24)$ corresponding to the modified forcing by $\tilde{R}^{(k)}$. In the domains where $\tilde{t}=0$, we can solve (22-24) analytically. These analytical solutions are used to form relations between the solution and its normal derivative, which must be satisfied by any solution that is bounded at infinity and fulfills the radiation condition (12). These relations are used as far-field boundary conditions. They are given by

$$
\begin{aligned}
& \tilde{R}^{(0)}=0, \frac{d \tilde{R}^{(0)}}{d x}=0, \quad x=-b, \\
& \tilde{R}^{(1)}=0, \frac{d \tilde{R}^{(1)}}{d x} 0, \quad x=-b, \\
& \frac{d \tilde{R}^{(k)}}{d x}-\sqrt{\kappa_{k}} \tilde{R}^{(k)} 0, \quad x=-b, k=2,3, \ldots, \\
& \frac{d \tilde{R}^{(k)}}{d x}+\sqrt{\kappa_{k}} \tilde{R}^{(k)} 0, \quad x=b, k=2,3, \ldots .
\end{aligned}
$$

The boundary conditions are exact in the sense that they do not affect the solution at all. The difference between $R^{(k)}$ and $\tilde{R}^{(k)}$, therefore, only depends on the difference between $t(x)$ and $\tilde{t}(x)$.

\section{Numerical RESUlts}

In this section we present numerical results for a second-order accurate discretization of (1-3). In $\S 5.1$ and $\S 5.2$ we comment on the numerical methods that were used to solve the subproblems. Thereafter, in $\S 5.3$, we study a number of test cases. We show that the iterative method converges rapidly, and that the convergence rate improves when the Froude number decreases. We also compare 
the solution with previous results obtained with a direct method [17] to indicate that the iteration converges to the correct solution. We verify numerically that the convergence rate is essentially independent of the grid size. It is demonstrated that the iterative method is efficient from a computational point of view; it requires less runtime than the direct solver already for two-dimensional problems. In addition, we show that the error committed by truncating the domain and introducing farfield boundary conditions decays exponentially with the size of the computational domain.

5.1. Solving the definite subproblem. We discretize the definite subproblem by second-order accurate finite differences on a composite overlapping grid. To apply the method, we divide the domain into simple overlapping subdomains and cover each subdomain with a component grid, see Figure 3. The subdomain close to the

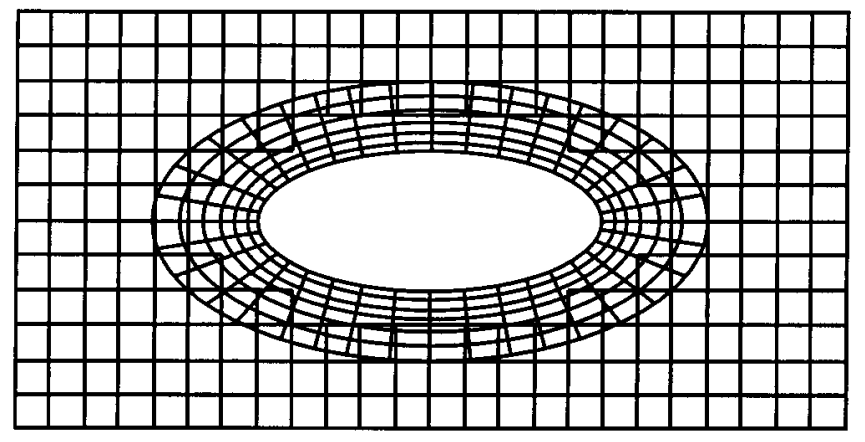

FiguRE 3. The composite overlapping grid

body is covered with a curvilinear grid and the surrounding sea is covered with a Cartesian grid. The main advantage with this method compared to discretizing the whole domain with one single grid is that each component grid can be made logically rectangular and without singularities. The grid functions on the component grids are coupled by continuity requirements, which are enforced by applying sufficiently accurate, in this case quadratic, interpolation relations between the grid functions at the interior boundaries where the component grids overlap. A comprehensive description of this approach for a similar problem is given in [17].

We use the program CMPGRD to construct the composite grids. Many aspects of composite grids and how to use this program are described in $[2,1,4]$. We would like to point out that this program is capable of constructing three-dimensional composite grids, so this method can also be used in three dimensions.

The resulting linear system of equations is solved by the YALE sparse matrix package [8]. This method requires of the order $\mathcal{O}\left(n^{2}\right)$ operations, where $n$ equals the number of grid points in the composite grid. However, for two-dimensional problems of moderate size, it turns out to be faster than multigrid or the conjugated gradient method, which are asymptotically faster.

5.2. Solving the indefinite subproblem. The number of terms in the series expansion (17), which equals the number of ordinary differential equations (2224) that must be solved, has to be limited in order to carry out the numerical calculation. We found by numerical experiments that it is sufficient to retain the first ten terms. This is related to the fact that the solution is smooth. 
We approximate the ordinary differential equations (22-24) by second-order accurate central differences. For simplicity, we use the same size of the computational domain and the same grid as for the definite subproblem. The tridiagonal systems of equations involved are solved by the subroutine DNBSL in the SLATEC package. The work needed to obtain a solution to the indefinite subproblem is of the order $\mathcal{O}\left(n_{1}\right)$, where $n_{1}$ is the product of the number of gridpoints in the discretization of one ordinary differential equation and the number of terms we retain in the series expansion.

5.3. Test runs. A circle with radius 0.1 was used as test body. The center of the circle was submerged 0.5 below the free surface and located at $x=0$, see Figure 4 . The Froude number was 0.4 unless otherwise stated. The cutoff function (65) had $\alpha=7 / 33$.

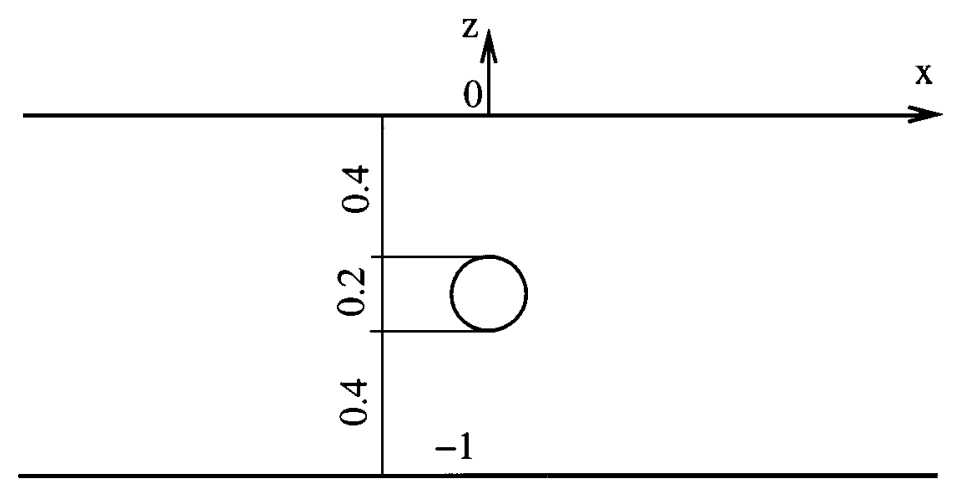

FIgure 4 . The test body; a circle with radius 0.1

To show an example of the solution, we present the surface elevation above the test body in Figure 5. We also show the perturbation potential in the whole computational domain in Figure 6.

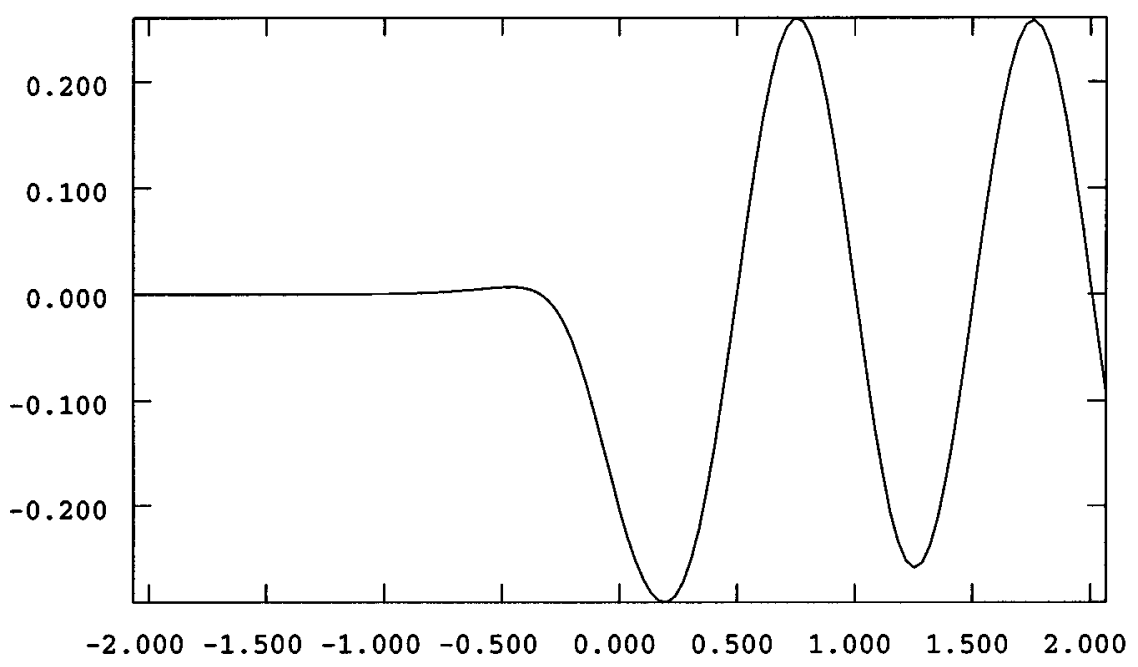

Figure 5 . The surface elevation above the test body, $\mu=0.4$ 


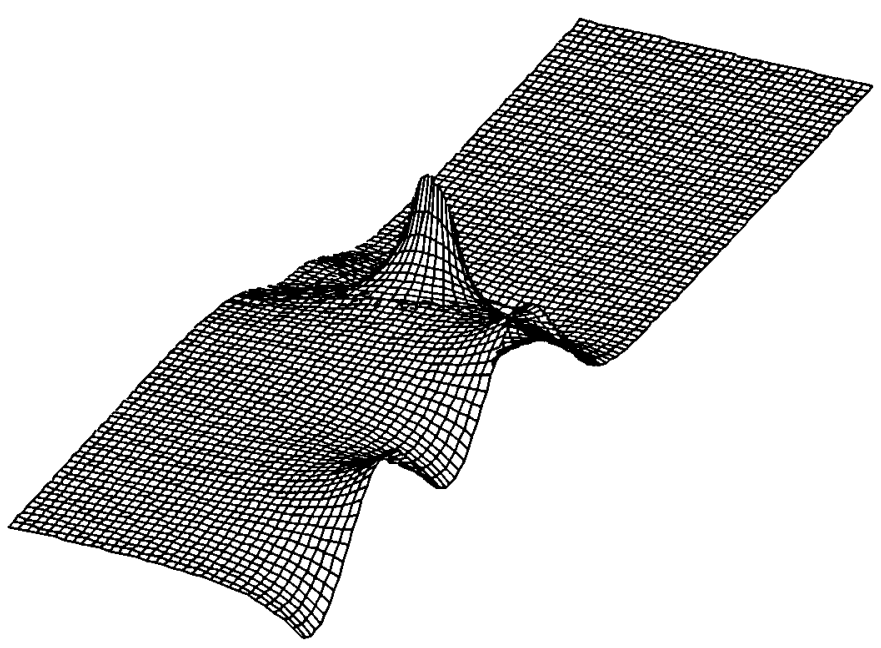

FiguRE 6 . The perturbation potential around the test body, $\mu=0.4$

In the following, we will compare solutions in the maximum norm over the part of the surface in the computational domain,

$$
|f|_{\infty, \text { sur }}=\sup _{-b<x<b}|f(x, 0)| .
$$

To find the order of accuracy of the iterative method, we compare solutions obtained with grid sizes $2 h, \sqrt{2} h$ and $h$. The corresponding solutions are denoted by $\phi_{2 h}, \phi_{\sqrt{2} h}$ and $\phi_{h}$, respectively. The length of the computational domain is 4.5 $(b=2.25)$. The number of grid points in the Cartesian grids is $17 \times 86,24 \times 132$ and $33 \times 172$, respectively, and in the grid around the body $17 \times 6,23 \times 8$ and $33 \times 11$, respectively. The results, which are presented in Table 1, indicate that the method is second-order accurate.

To ensure that the iteration converges to the right solution, we compare the iterative solution with the solution computed by the direct method described in [17]. We cannot expect perfect agreement, because even though both methods are secondorder accurate, they correspond to different sets of discrete approximations. Furthermore, the far-field boundary conditions are not the same in the two approaches. Hence, the difference between the solutions will only tend to zero as $h^{2}$ if the computational domain is sufficiently large, so that effects from the far-field boundaries can be neglected. We use the same grids as described above. The solutions obtained with the direct solver are denoted $\phi_{2 h}^{d}, \phi_{\sqrt{2} h}^{d}$ and $\phi_{h}^{d}$, respectively. The results, cf. Table 2 , show that the difference between the solutions corresponding to the two methods tends to zero approximately as $h^{2}$. The cpu-time required for solving these problems on a Sun $4 / 20$ with 8 megabyte memory can be found in Table 3 .

Next, we study the convergence rate of the iterative method. In Tables 4-9 we present $\left|\phi^{(k)}-\phi^{(k-1)}\right|_{\infty, \text { sur }} /|\phi|_{\infty, \text { sur }}$ as function of $k$ for different values of $\mu$ and for

TABLE 1. Comparison between solutions computed with different grid sizes

\begin{tabular}{l|l}
\hline$\left|\phi_{2 h}-\phi_{h}\right|_{\infty, \text { sur }}$ & $\left|\phi_{\sqrt{2} h}-\phi_{h}\right|_{\infty, \text { sur }}$ \\
\hline $1.146 \times 10^{-2}$ & $4.609 \times 10^{-3}$ \\
\hline
\end{tabular}


TABle 2. Comparison between solutions computed with the iterative method and the direct method for different grid sizes

\begin{tabular}{l|l|l}
\hline$\left|\phi_{2 h}^{d}-\phi_{2 h}\right|_{\infty, \text { sur }}$ & $\left|\phi_{\sqrt{2} h}^{d}-\phi_{\sqrt{2} h}\right|_{\infty, \text { sur }}$ & $\left|\phi_{h}^{d}-\phi_{h}\right|_{\infty, \text { sur }}$ \\
\hline $4.795 \times 10^{-3}$ & $2.497 \times 10^{-3}$ & $1.343 \times 10^{-3}$ \\
\hline
\end{tabular}

TABLE 3. Cpu-time comparison between the iterative method and the direct method (seconds)

\begin{tabular}{l|l|l|l}
\hline & $2 h$ & $\sqrt{2} h$ & $h$ \\
\hline \# of equations & 1178 & 2202 & 4390 \\
\hline iterative & 13.1 & 25.1 & 53.9 \\
\hline direct & 16.6 & 102.4 & 826 \\
\hline
\end{tabular}

TABLE 4. Convergence rate, $\mu=0.8$

\begin{tabular}{l|l|l|l}
\hline iteration & $\phi_{2 h}$ & $\phi_{\sqrt{2} h}$ & $\phi_{h}$ \\
\hline 1 & $9.336 \times 10^{-1}$ & $9.264 \times 10^{-1}$ & $9.216 \times 10^{-1}$ \\
\hline 2 & $5.835 \times 10^{-2}$ & $6.283 \times 10^{-2}$ & $7.398 \times 10^{-2}$ \\
\hline 3 & $9.789 \times 10^{-3}$ & $1.097 \times 10^{-2}$ & $4.748 \times 10^{-3}$ \\
\hline 4 & $9.344 \times 10^{-4}$ & $5.336 \times 10^{-3}$ & $5.336 \times 10^{-4}$ \\
\hline
\end{tabular}

TABle 5. Convergence rate, $\mu=0.7$

\begin{tabular}{l|l|l|l}
\hline iteration & $\phi_{2 h}$ & $\phi_{\sqrt{2} h}$ & $\phi_{h}$ \\
\hline 1 & $9.502 \times 10^{-1}$ & $9.547 \times 10^{-1}$ & $9.554 \times 10^{-1}$ \\
\hline 2 & $5.509 \times 10^{-2}$ & $5.083 \times 10^{-2}$ & $4.879 \times 10^{-2}$ \\
\hline 3 & $3.756 \times 10^{-3}$ & $3.306 \times 10^{-3}$ & $3.174 \times 10^{-3}$ \\
\hline 4 & $3.447 \times 10^{-4}$ & $4.883 \times 10^{-4}$ & $1.207 \times 10^{-3}$ \\
\hline 5 & $*$ & $*$ & $7.231 \times 10^{-4}$ \\
\hline
\end{tabular}

TABLE 6. Convergence rate, $\mu=0.6$

\begin{tabular}{l|l|l|l}
\hline iteration & $\phi_{2 h}$ & $\phi_{\sqrt{2} h}$ & $\phi_{h}$ \\
\hline 1 & $9.561 \times 10^{-1}$ & $9.619 \times 10^{-1}$ & $9.561 \times 10^{-1}$ \\
\hline 2 & $4.008 \times 10^{-2}$ & $4.361 \times 10^{-2}$ & $4.910 \times 10^{-2}$ \\
\hline 3 & $2.209 \times 10^{-3}$ & $2.597 \times 10^{-3}$ & $3.211 \times 10^{-3}$ \\
\hline 4 & $1.541 \times 10^{-4}$ & $1.029 \times 10^{-4}$ & $1.462 \times 10^{-4}$ \\
\hline
\end{tabular}

different grid sizes. The iteration is truncated when this quantity becomes less than $10^{-3}$. The results confirm that the convergence rate improves when $\mu$ decreases and that it is essentially independent of the grid size.

In order to examine the effect of the far-field boundary conditions in the definite subproblem and the truncation of the forcing function $t(x)$ in the indefinite subproblem, we study how the solution depends on the size of the computational domain. We consider the lengths $1.2,3.2$ and 5.2, i.e., $b=0.6,1.6,2.6$, respectively, and we denote the solutions on these grids by $\phi^{1.2}, \phi^{3.2}$ and $\phi^{5.2}$, respectively. The 
TABLE 7. Convergence rate, $\mu=0.5$

\begin{tabular}{l|l|l|l}
\hline iteration & $\phi_{2 h}$ & $\phi_{\sqrt{2} h}$ & $\phi_{h}$ \\
\hline 1 & $9.468 \times 10^{-1}$ & $9.540 \times 10^{-1}$ & $9.588 \times 10^{-1}$ \\
\hline 2 & $4.965 \times 10^{-2}$ & $4.351 \times 10^{-2}$ & $3.769 \times 10^{-2}$ \\
\hline 3 & $5.370 \times 10^{-3}$ & $4.544 \times 10^{-3}$ & $3.860 \times 10^{-3}$ \\
\hline 4 & $1.810 \times 10^{-4}$ & $3.459 \times 10^{-4}$ & $6.479 \times 10^{-4}$ \\
\hline
\end{tabular}

TABLE 8. Convergence rate, $\mu=0.4$

\begin{tabular}{l|l|l|l}
\hline iteration & $\phi_{2 h}$ & $\phi_{\sqrt{2} h}$ & $\phi_{h}$ \\
\hline 1 & $9.737 \times 10^{-1}$ & $9.764 \times 10^{-1}$ & $9.783 \times 10^{-1}$ \\
\hline 2 & $2.662 \times 10^{-2}$ & $2.347 \times 10^{-2}$ & $2.164 \times 10^{-2}$ \\
\hline 3 & $6.189 \times 10^{-4}$ & $3.799 \times 10^{-4}$ & $2.982 \times 10^{-4}$ \\
\hline
\end{tabular}

TABle 9. Convergence rate, $\mu=0.3$

\begin{tabular}{l|l|l|l}
\hline iteration & $\phi_{2 h}$ & $\phi_{\sqrt{2} h}$ & $\phi_{h}$ \\
\hline 1 & $9.905 \times 10^{-1}$ & $9.913 \times 10^{-1}$ & $9.916 \times 10^{-1}$ \\
\hline 2 & $9.861 \times 10^{-3}$ & $9.022 \times 10^{-3}$ & $8.111 \times 10^{-3}$ \\
\hline 3 & $6.724 \times 10^{-5}$ & $2.556 \times 10^{-4}$ & $7.020 \times 10^{-4}$ \\
\hline
\end{tabular}

TABLE 10. Comparison between solutions corresponding to different lengths of the computational domain

\begin{tabular}{l|l}
\hline$\left|\phi^{5.2}-\phi^{1.2}\right|_{\infty, \text { sur }}$ & $\left|\phi^{5.2}-\phi^{3.2}\right|_{\infty, \text { sur }}$ \\
\hline $8.455 \times 10^{-3}$ & $5.220 \times 10^{-4}$ \\
\hline
\end{tabular}

grid size in both directions of the the Cartesian grid was $1 / 33$. We used $33 \times 11$ grid points in the grid around the body. The results, given in Table 10, suggest that the influence of the far-field boundary conditions decays exponentially with the length of the computational domain.

\section{Acknowledgment}

Professor H.-O. Kreiss is gratefully acknowledged for many inspiring discussions on the present problem. We would also like to thank Dr. D. L. Brown and Dr. G. Chesshire for sharing their thorough knowledge on composite overlapping grids.

\section{REFERENCES}

1. D. L. Brown, G. Chesshire, and W. D. Henshaw, Getting started with CMPGRD. Introductory user's guide and reference manual. LA-UR 90-3729, Los Alamos National Laboratory, 1989.

2. D. L. Brown, G. Chesshire, and W. D. Henshaw, An explanation of the CMPGRD composite grid data structure. IBM Research Report RC 14354, IBM Research Division, Yorktown Heights, NY, 1990.

3. J. Cahouet and M. Lenoir, Résolution numérique du problème non linéaire de la résistance de vagues bidimensionelle. C. R. Acad. Sc. Paris II, 297:819-822, 1983. MR 86j:76009 
4. G. Chesshire and W. D. Henshaw, Composite overlapping meshes for the solution of partial differential equations. J. Comput. Phys., 90(1):1-64, 1990. MR 91f:76043

5. R. Courant and D. Hilbert, Methods of Mathematical Physics, vol II. Interscience Publishers, New York, 1962. MR 25:4216

6. C. W. Dawson, A practical computer method for solving ship wave problems. In Proceedings of the Second International Conference on Numerical Ship Hydrodynamics, pages 30-38. University of California, Berkeley, 1977.

7. L. J. Doctors and R. F. Beck, Convergence properties of the Neumann-Kelvin problem for a submerged body. J. Ship Res., 31:227-234, 1987.

8. S. Eisenstat, M. Gursky, M. H. Schultz, and A. H. Sherman, The Yale matrix package II: The non-symmetric case. Report 114, Dept. of Computer Science, Yale University, 1977.

9. P. S. Jensen, On the numerical radiation condition in the steady state ship wave problem. $J$. Ship Res., 31:14-22, 1987.

10. M. Lenoir and A. Tounsi, The localized finite element method and its application to the twodimensional sea-keeping problem. SIAM J. Numer. Anal., 25:729-752, 1988. MR 89k:65138

11. W. Lindemuth, T. J. Ratcliffe, and A. M. Reed, SHD 1260-1, David W. Taylor Naval Ship Research \& Development Center, 1988.

12. J. F. Malmliden, An efficient numerical method for 3-D flow around a submerged body. TRITA-NA 9306, Department of Numerical Analysis and Computing Science, Royal Institute of Technology, 1993.

13. C. C. Mei and H. S. Chen, A hybrid element method for steady linearized free-surface flows. Int. J. Numer. Meth. Eng., 10:1153-1175, 1976.

14. D. E. Nakos and P. D. Sclavounos, On steady and unsteady ship wave patterns. J. Fluid Mech., 215:263-288, 1990. MR 91c:76022

15. J. N. Newman, Evaluation of the wave-resistance Green function: Part 2. The single integral on the centerplane. J. Ship Res., 31(3):145-150, 1987.

16. N. A. Petersson, A numerical method to calculate the two-dimensional flow around an underwater obstacle. SIAM J. Numer. Anal., 29:20-31, 1992. MR 92j:76010

17. N. A. Petersson and J. F. Malmliden, Computing the flow around a submerged body using composite grids. J. Comput. Phys., 105:47-57, 1993.

18. G. B. Whitham, Linear and nonlinear waves. Wiley-Interscience, New York, 1974. MR 58:3905

19. G. X. Wu and R. E. Taylor, Hydrodynamic forces on submerged oscillating cylinders at forward speed. Proc. R. Soc. Lond., A 414:149-170, 1987.

20. F. Xia and L. Larsson, A calculation method for the lifting potential flow around yawed surface piercing 3-D bodies. In Proceedings of the 16'th Symposium on Naval Hydrodynamics, 1986, pp. 583-597.

Center for Computational Mathematics and Mechanics, Royal Institute of TechNology, 10044 Stockholm, Sweden

E-mail address: johanm@prosolvia.se

Department of Mathematics, University of California, Los Angeles, California 90024

E-mail address: andersp@na.chalmers.se 